九州大学学術情報リポジトリ

Kyushu University Institutional Repository

\title{
RELATIONAL ASPECTS OF RELATIONAL DATABASE DEPENDENCIES
}

Okuma, Hitomi

Department of Informatics, Kyushu University

Kawahara, Yasuo

Department of Informatics, Kyushu University

https://doi.org/10.5109/13495

出版情報: Bulletin of informatics and cybernetics. 32 (2)，pp.91-104，2000-12. 統計科学研究会 バージョン：

権利関係 : 


\title{
RELATIONAL ASPECTS OF RELATIONAL DATABASE DEPENDENCIES
}

\author{
By \\ Hitomi OKUMA ${ }^{*}$ and Yasuo KaWahara ${ }^{\dagger}$
}

\begin{abstract}
This paper presents a relational treatment of inference rules for functional and multivalued dependencies in relational databases, to show the soundness and the completeness of the inference rules in Dedekind categories, which also cover the fuzzy case.
\end{abstract}

\section{Introduction}

It is well-known that relational database models were introduced by Codd (1970). Functional and multivalued dependencies are the most important examples of relationships (constraints) between attributes in database relations. The completeness theorems for functional and multivalued dependencies have been proved by Armstrong (1974) and Beeri et al. (1977), respectively.

Schmidt and Ströhlein (1993) explained a basic relational feature of functional dependency for relational models of databases, and Ounalli and Jaoua (1997) studied difunctional dependencies in relational databases. Orlowska (1987) proposed a relational formulation of functional; multivalued and other dependencies, and an axiomatic relational calculus for dependency theory was developed in Buszkowski and Orlowska (1998). MacCaull (2000a), (2000b) gave a relational formulation for functional, multivalued dependencies and association rules, and developed a Rasiowa/Sikorski-style tableaux method of proof which is sound and complete for the implication problem for these dependencies.

In this paper we will propose a foundation of a relational treatment for functional and multivalued dependencies in Dedekind categories.

This paper is organized as follows. In Section 2 we review the definitions of Dedekind categories (Olivier and Serrato (1980) and Kawahara (1998)) as one of relational categories, and relational products (Desharnais (1997)), that provide total spaces of database tuples. In section 3 we introduce a notion of database schemes to present a relational and algebraic framework to study database dependencies. In Sections 4 and 5 we will give the definitions of functional and multivalued dependencies in our database schemes, and show the inference rules, which implies the soundness theorems for the dependencies. Finally we will remark that database schemes with a weaker condition have sufficient basic properties to show the completeness theorems.

\footnotetext{
* Department of Informatics, Kyushu University 33 Fukuoka 812-8581 Japan. okuma@i.kyushu-u.ac.jp

$\dagger$ Department of Informatics, Kyushu University 33 Fukuoka 812-8581 Japan. kawahara@i.kyushuu.ac.jp
} 


\section{Relational Products}

In this section we recall the definition of Dedekind categories discussed in Olivier and Serrato (1980) and Kawahara (1998), and review relational products (Desharnais (1997)) in Dedekind categories.

Throughout this paper, a morphism $\alpha$ from an object $X$ into an object $Y$ in a Dedekind category (which will be defined below) will be denoted by a half arrow $\alpha$ : $X \rightarrow Y$, and the composite of a morphism $\alpha: X \rightarrow Y$ followed by a morphism $\beta: Y \neg Z$ will be written as $\alpha \beta: X \rightarrow Z$. Also we will denote the identity morphism on $X$ as id ${ }_{X}$.

DEFINITION 2.1. A Dedekind category $\mathcal{D}$ is a category satisfying the following:

D1. [Complete Heyting Algebra] For all pairs of objects $X$ and $Y$ the hom-set $\mathcal{D}(X, Y)$ consisting of all morphisms of $X$ into $Y$ is a complete Heyting algebra (namely, a complete distributive lattice) with the least morphism $0_{X Y}$ and the greatest morphism $\nabla_{X Y}$. Its algebraic structure will be denoted by

$$
\mathcal{D}(X, Y)=\left(\mathcal{D}(X, Y), \sqsubseteq, \sqcup, \sqcap, 0_{X Y}, \nabla_{X Y}\right)
$$

That is,

(a) $\sqsubseteq$ is a partial order on $\mathcal{D}(X, Y)$,

(b) $\forall \alpha \in \mathcal{D}(X, Y):: 0_{X Y} \sqsubseteq \alpha \sqsubseteq \nabla_{X Y}$,

(c) $\sqcup_{i \in I} \alpha_{i} \sqsubseteq \alpha$ iff $\alpha_{i} \sqsubseteq \alpha$ for all $i \in I$,

(d) $\alpha \sqsubseteq \Pi_{i \in I} \alpha_{i}$ iff $\alpha \sqsubseteq \alpha_{i}$ for all $i \in I$,

(e) $\alpha \Pi\left(\sqcup_{i \in I} \alpha_{i}\right)=\sqcup_{i \in I}\left(\alpha \Pi \alpha_{i}\right)$.

D2. [Converse] There is given a converse operation ${ }^{\sharp} \mathcal{D}(X, Y) \rightarrow \mathcal{D}(Y, X)$. That is, for all morphisms $\alpha, \alpha^{\prime}: X \rightarrow Y, \beta: Y \rightarrow Z$, the following involutive laws hold:

(a) $(\alpha \beta)^{\sharp}=\beta^{\sharp} \alpha^{\sharp}$,

(b) $\left(\alpha^{\sharp}\right)^{\sharp}=\alpha$,

(c) If $\alpha \sqsubseteq \alpha^{\prime}$, then $\alpha^{\natural} \sqsubseteq \alpha^{\prime \prime}$.

D3. [Dedekind Formula] For all morphisms $\alpha: X \rightarrow Y, \beta: Y \rightarrow Z$ and $\gamma: X \rightarrow Z$ the Dedekind formula $\alpha \beta \sqcap \gamma \sqsubseteq \alpha\left(\beta \sqcap \alpha^{\mathrm{H}} \gamma\right)$ holds.

D4. [Residue] For all morphisms $\beta: Y-Z$ and $\gamma: X \rightarrow Z$ the residue (or division, weakest precondition) $\gamma \div \beta: X \neg Y$ is a morphism such that $\alpha \beta \sqsubseteq \gamma$ if and only if $\alpha \sqsubseteq \gamma \div \beta$ for all morphisms $\alpha: X \rightarrow Y$.

A morphism $f: X \rightarrow Y$ such that $f^{\sharp} f \sqsubseteq$ id $_{Y}$ is called a partial function (univalent) and may be introduced as $f: X \rightarrow Y$. A partial function $f: X \rightarrow Y$ such that $\operatorname{id}_{X} \sqsubseteq f f^{\sharp}$ (total) is called a function. A morphism $\alpha: X \neg Y$ such that $\alpha \alpha^{\sharp} \alpha \sqsubseteq \alpha$ is difunctional. In what follows the word relation is a synonym for morphism of a Dedekind category.

An object $I$ of a Dedekind category $\mathcal{D}$ is called a unit if $0_{I I} \neq \mathrm{id}_{I}=\nabla_{I I}$. A unit $I$ of $\mathcal{D}$ is called strict if $\nabla_{X I} \nabla_{I X}=\nabla_{X X}$ for all objects $X$ of $\mathcal{D}$. An $I$-point $x$ of $X$ is a function $x: I \rightarrow X$, that is, a univalent and total relation.

For the basic properties of Dedekind categories, or relation categories, the reader is referred to Freyd and Ščedrov (1990), Schmidt and Ströhlein (1993). 
Lemma 2.2. Let $\alpha: X \rightarrow Y$ be a relation, and let $x: I \rightarrow X$ and $y: I \rightarrow Y$ be $I$-points of $X$ and $Y$, respectively. Then

(a) $x^{\sharp} y \sqsubseteq \alpha$ if and only if $x \alpha y^{\sharp}=\mathrm{id}_{I}$,

(b) If $x^{\sharp} y \cap \alpha=0_{X Y}$, then $x \alpha y^{\sharp}=0_{I I}$.

Proof. (a) Assume that $x^{\sharp} y \sqsubseteq \alpha$. Then id I $_{I} \sqsubseteq x x^{\sharp} y y^{\sharp} \sqsubseteq x \alpha y^{\sharp}$ by the totality of $x$ and $y$. Hence $x \alpha y^{\sharp}=\mathrm{id}_{I}$. For the converse, suppose that $x \alpha y^{\sharp}=\mathrm{id}_{I}$. Then $x^{\sharp} y=x^{\sharp} x \alpha y^{\sharp} y \sqsubseteq \alpha$, since $x$ and $y$ are univalent.

(b) Assume that $x^{\sharp} y \sqcap \alpha=0_{X Y}$. Then $x \alpha y^{\sharp}=x \alpha y^{\sharp} \sqcap \mathrm{id}_{I} \sqsubseteq x\left(\alpha \sqcap x^{\sharp} y\right) y^{\sharp}=0_{I I}$.

Let $U$ be a finite set and let $\left\{D_{a} \mid a \in U\right\}$ be a $U$-indexed set of objects in a Dedekind category $\mathcal{D}$. We call $U$ a set of attributes and $D_{a}$ a domain of possible $a$-values for an attribute $a \in U$. In this section we assume that the Dedekind category $\mathcal{D}$ has finite relational products for all subsets of $\left\{D_{\mathfrak{a}} \mid a \in U\right\}$. That is, for each subset $X \subseteq U$ there exists an object $T[X]$ of $\mathcal{D}$ and an $X$-indexed set $\left\{p_{X a}: T[X] \rightarrow D_{a} \mid a \in X\right\}$ of functions (called projections) in $\mathcal{D}$ satisfying the following three conditions:

(PD1) $\forall a \in X:: p_{X a}^{\sharp} p_{X a}=\mathrm{id}_{D_{a}}$,

(PD2) $\forall a \in X:: p_{X a}^{\sharp}\left(\Pi_{b \neq a} p_{X b} p_{X b}^{\sharp}\right)=\nabla_{D_{a} T[X]}$,

(PD3) $\Pi_{a \in X} p_{X a} p_{X a}^{\sharp}=\mathrm{id}_{T[X]}$.

REMARK. (i) In the case that $X$ is a singleton set, namely, $X=\{a\}$, we set $T[X]=D_{\mathfrak{a}}$ and $p_{\{a\} a}=\mathrm{id}_{D_{\mathfrak{a}}}$. Then the conditions (PD1), (PD2) and (PD3) hold under the convention that the infimum of the empty set of relations is the universal relation. (ii) In the case that $X$ is the empty set, we set $T[\emptyset]=I$. Then the above conditions are valid, too.

THEOREM 2.3. (Sharpness) Let $Y$ be a subset of $U$, and let $\left\{\alpha_{y}: A \rightarrow D_{y} \mid y \in Y\right\}$ and $\left\{\beta_{y}: B \rightarrow D_{y} \mid y \in Y\right\}$ be $Y$-indexed sets of relations in $\mathcal{D}$. If all $\alpha_{y}$ are difunctional, or all $\beta_{y}$ are difunctional, then

$$
\Pi_{y \in Y} \alpha_{y} \beta_{y}^{\sharp}=\left(\Pi_{y \in Y} \alpha_{y} p_{Y y}^{\sharp}\right)\left(\Pi_{y \in Y} p_{Y y} \beta_{y}^{\sharp}\right) .
$$

For the proof of this theorem the reader is referred to Desharnais (1997).

For a subset $Y$ of $X$ we define a relation $p_{X Y}: T[X] \rightarrow T[Y]$ as follows:

$$
p_{X Y}=\Pi_{\mathfrak{a} \in Y} p_{X \mathfrak{a}} p_{Y \mathfrak{a}}^{\sharp} \text {. }
$$

It is trivial that $p_{X\{a\}}=p_{X a}$ for all $a$ in $X$, and $p_{X X}=\mathrm{id}_{T[X]}$.

Proposition 2.4. Let $X, Y$ and $Z$ be subsets of $U$. Then the following hold:

(a) The relation $p_{X Y}: T[X] \rightarrow T[Y]$ is the unique function such that $p_{X Y} p_{Y a}=p_{X a}$ for all $a \in Y$,

(b) $p_{X Y} p_{Y Z}=p_{X Z}$ if $Z \subseteq Y \subseteq X$. 
Proof. (a) First we will see that $p_{X Y}$ is univalent and total. Univalence follows from

$$
\begin{array}{rlrl}
p_{X Y}^{\Downarrow} p_{X Y} & =\left(\Pi_{a \in Y} p_{Y a} p_{X a}^{\sharp}\right)\left(\Pi_{a \in Y} p_{X a} p_{Y a}^{\sharp}\right) & \\
& \sqsubseteq \Pi_{a \in Y} p_{Y a} p_{X a}^{\sharp} p_{X a} p_{Y a}^{\sharp} & & \{\text { Sub-distributivity }\} \\
& \sqsubseteq \Pi_{a \in Y} p_{Y a} p_{Y a}^{\sharp} & & \left\{p_{X a}^{\sharp} p_{X a} \sqsubseteq \mathrm{id}_{D_{a}}\right\} \\
& =\operatorname{id}_{T[Y]} & & \{(\mathrm{PD} 3)\},
\end{array}
$$

and totality from

$$
\begin{aligned}
\mathrm{id}_{T[X]} & \sqsubseteq \Pi_{a \in Y} p_{X a} p_{X_{a}}^{\sharp} & & \left\{\operatorname{id}_{T[X]} \sqsubseteq p_{X a} p_{X a}^{\sharp}\right\} \\
& =\left(\Pi_{a \in Y} p_{X a} p_{Y a}^{\sharp}\right)\left(\Pi_{a \in Y} p_{Y a} p_{X a}^{\sharp}\right) & & \text { Theorem 2.3 (sharpness) }\} \\
& =p_{X Y p_{X Y}}^{\sharp}, & &
\end{aligned}
$$

since all $p_{X a}$ are difunctional. Finally assume that $f: T[X] \rightarrow T[Y]$ is a function such that $f p_{Y a}=p_{X a}$ for all $a \in Y$. Then

$$
f=f \mathrm{id}_{T[Y]}=f\left(\Pi_{a \in Y} p_{Y a} p_{Y a}^{\sharp}\right)=\Pi_{a \in Y} f p_{Y_{a}} p_{Y a}^{\sharp}=\Pi_{a \in Y} p_{X a} p_{Y a}^{\sharp}=p_{X Y},
$$

which proves the uniqueness of $p_{X Y}$.

(b) It is easy to see that $p_{X Y} p_{Y Z} p_{Z a}=p_{X Y} p_{Y a}=p_{X a}=p_{X Z} p_{Z a}$ for all $a \in Z$, and so $p_{X Y} p_{Y Z}=p_{X Z}$ by the uniqueness of $p_{X Z}$, proved in (a).

For each subset $X$ of $U$ we define an equivalence relation $\theta[X]: T[U] \rightarrow T[U]$ by $\theta[X]=\Pi_{a \in X} p_{U a} p_{U a}^{\sharp}$. When $X$ is empty set, set $\theta[\emptyset]=\nabla_{T[U] T[U]}$.

Proposition 2.5. Let $X$ and $Y$ be subsets of $U$. Then

(a) $\theta[U]=\mathrm{id}_{T[U]}$ and $\theta[X]=p_{U X} p_{U X}^{\sharp}$,

(b) $\theta[X \cup Y]=\theta[X] \cap \theta[Y]$,

(c) $\theta[X \cap Y]=\theta[X] \theta[Y]$.

Proof. (a) First note that $\Pi_{a \in X} p_{X a} p_{X a}^{\sharp}=\mathrm{id}_{T[X]}$ by (PD3), and that $p_{U X} p_{X a}=$ $p_{U a}$ for all $a \in X$ by Proposition 2.4(a). We have

$$
\begin{array}{rlrl}
p_{U X X} p_{U X}^{\sharp} & =p_{U X}\left(\Pi_{a \in X} p_{X a} p_{X a}^{\sharp}\right) p_{U X}^{\sharp} & \left\{\Pi_{a \in X} p_{X a} p_{X a}^{\sharp}=\mathrm{id}_{T[X]}\right\} \\
& =\Pi_{a \in X} p_{U X} p_{X a} p_{X a}^{\sharp} p_{U X}^{\sharp} & \\
& =\Pi_{a \in X} p_{U a} p_{U a}^{\sharp} & & \left\{p_{U X X} p_{X a}=p_{U a}\right\} \\
& =\theta[X] . & &
\end{array}
$$

(b) From the definition we have $\theta[X] \sqcap \theta[Y]=\left(\Pi_{\alpha \in X} p_{U_{a}} p_{U_{a}}^{\sharp}\right) \sqcap\left(\Pi_{\alpha \in Y} p_{U_{a}} p_{U_{a}}^{\sharp}\right)=$ $\Pi_{a \in X \cup Y} p_{U a} p_{U a}^{\sharp}=\theta[X \cup Y]$.

(c) For a subset $X$ of $U$ define a $U$-indexed set $\left\{\alpha_{a}^{X} \mid a \in U\right\}$ of relations by $\alpha_{a}^{X}=$ id $_{D_{a}}$ if $a \in X$ and $\alpha_{a}^{X}=\nabla_{D_{a} D_{a}}$ otherwise. As $p_{U a} \alpha_{a}^{X} p_{U a}^{\sharp}=p_{U a} \nabla_{D_{a} D_{a}} p_{U a}^{\sharp}=\nabla_{T[U] T[U]}$ for $a \notin X$ we have $\Pi_{a \in U} p_{U a} \alpha_{a}^{X} p_{U a}^{\sharp}=\Pi_{a \in X} p_{U a} p_{U a}^{\sharp}=\theta[X]$. Therefore, applying sharpness (Theorem 2.3) we obtain

$$
\begin{aligned}
\theta[X] \theta[Y] & =\left(\Pi_{a \in U} p_{U a} \alpha_{a}^{X} p_{U a}^{\sharp}\right)\left(\Pi_{a \in U} p_{U a} \alpha_{\mathfrak{a}}^{Y} p_{U a}^{\sharp}\right) & & \\
& =\Pi_{a \in U} p_{U a} \alpha_{a}^{X} \alpha_{a}^{Y} p_{U \mathfrak{a}}^{\sharp} & & \{\text { Theorem 2.3 (sharpness) }\} \\
& =\Pi_{a \in U} p_{U a} \alpha_{a}^{X \cap Y} p_{U a}^{\sharp} & & \left\{\alpha_{a}^{X} \alpha_{a}^{Y}=\alpha_{a}^{X \cap Y}\right\} \\
& =\theta[X \cap Y] . & &
\end{aligned}
$$


(Note that all $p_{U a} \alpha_{a}^{X}$ are difunctional.)

Throughout this paper we will assume that $\mathcal{D}$ is a fixed Dedekind category with a strict unit $I$ and relational products.

\section{Attribute Schemes}

To realize a relational treatment of some dependencies on relational databases we will introduce a notion of attribute schemes in the following:

Definition 3.1. An attribute scheme in a Dedekind category $\mathcal{D}$ is a triple $(U, T, \theta)$ of a set $U$ of attributes, an object $T$ of $\mathcal{D}$ and a mapping $\theta: \wp(U) \rightarrow \mathcal{D}(T, T)$ assigning a relation $\theta[X]$ on $T$ to each subset $X$ of $U$ such that

(a) $\theta[X]$ is an equivalence relation for each subset $X$ of $U$,

(b) $\theta[U]=\mathrm{id}_{T}$ and $\theta[\theta]=\nabla_{T T}$,

(c) $\theta[X \cup Y]=\theta[X] \cap \theta[Y]$ and $\theta[X \cap Y]=\theta[X] \theta[Y]$ for all subsets $X$ and $Y$ of $U$.

Note that $\theta[X]$ is an equivalence relation if and only if it is reflexive (id $T \subseteq \theta[X]$ ), symmetric $\left(\theta[X]^{\sharp} \sqsubseteq \theta[X]\right)$ and transitive $(\theta[X] \theta[X] \subseteq \theta[X])$.

Definition 3.2. A database relation on an attribute scheme $(U, T, \theta)$ is a relation $r: T \rightarrow T$ such that $r \sqsubseteq \mathrm{id}_{T}$.

The following shows technical properties of database relations in attribute schemes.

LEMMA 3.3. Let $r$ be a database relation on an attribute scheme $(U, T, \theta)$. For all subsets $X, Y$ and $W$ of $U$ the following hold:

(a) If $Y \subseteq X$, then $\theta[X] \subseteq \theta[Y]$,

(b) If $X \cup Y=U$, then $\theta[X] r \theta[Y] \sqcap \theta[W]=\theta[X \cup W] r \theta[Y \cup W] \sqcap \theta[W]$.

Proof. (a) Assume that $Y \subseteq X$. Then $\theta[X]=\theta[X \cup Y]=\theta[X] \sqcap \theta[Y] \sqsubseteq \theta[Y]$ (and $\theta[X] \theta[Y]=\theta[X \cap Y]=\theta[Y]$ ).

(b) It is clear from (a) that $\theta[X \cup W] r \theta[Y \cup W] \sqcap \theta[W] \sqsubseteq \theta[X] r \theta[Y] \sqcap \theta[W]$. Conversely we have

$$
\begin{aligned}
& \theta[X] r \theta[Y] \sqcap \theta[W] \\
& \sqsubseteq\left\{\theta[X] r \sqcap \theta[W](\theta[Y])^{\sharp}\right\}\left\{\theta[Y] \sqcap(\theta[X] r)^{\mathbf{t}} \theta[W]\right\} \\
& \sqsubseteq\left\{\theta[X] \sqcap \theta[W](\theta[Y])^{\mathfrak{t}} r^{\sharp}\right\} r\left\{\theta[Y] \sqcap(\theta[X] r)^{\sharp} \theta[W]\right\} \\
& \text { ᄃ }(\theta[X] \sqcap \theta[W] \theta[Y]) r(\theta[Y] \sqcap \theta[X] \theta[W]) \\
& =\theta[X \cup(W \cap Y)] r \theta[Y \cup(X \cap W)] \\
& =\theta[X \cup W] r \theta[Y \cup W] \\
& \{X \cup(W \cap Y)=X \cup W, Y \cup(X \cap W)=Y \cup W \text { by } X \cup Y=U\} \text {. }
\end{aligned}
$$

Proposition 3.4. Let $r$ be a database relation on an attribute scheme $(U, T, \theta)$. For all subsets $X$ and $Y$ of $U$ the following hold: 
(a) $r \theta[X] r \sqsubseteq \theta[Y]$ if and only if $r \theta[X] r \sqsubseteq r \theta[Y] r$.

(b) $\theta[\neg Y] r \theta[Y] \sqcap \theta[X]=\theta[X \cup \neg Y] r \theta[X \cup Y] \cap \theta[X]=\theta[\neg(X \cup Y)] r \theta[X \cup Y] \sqcap \theta[X]$.

Proof. (a) It is obvious from $r \sqsubseteq \mathrm{id}_{T}$.

(b) From Lemma 3.3(b) we have

$$
\begin{aligned}
\theta[\neg(X \cup Y)] r \theta[X \cup Y] \sqcap \theta[X] & =\theta[X \cup \neg(X \cup Y)] r \theta[X \cup Y] \sqcap \theta[X] \\
& =\theta[X \cup \neg Y] r \theta[X \cup Y] \sqcap \theta[X] .
\end{aligned}
$$

\section{Functional Dependencies}

Let $r$ be a database relation on an attribute scheme $(U, T[U], \theta)$ discussed in Section 2. A relation $\alpha_{X Y}=p_{U X}^{\sharp} r p_{U Y}: T[X]-T[Y]$ is functional (or, univalent) if and only if :

$$
\begin{array}{lll} 
& \alpha_{X Y}^{\sharp} \alpha_{X Y} \sqsubseteq \mathrm{id}_{T[Y]} & \\
\Longleftrightarrow \quad\left(p_{U X}^{\sharp} r p_{U Y}\right)^{\sharp}\left(p_{U X}^{\sharp} r p_{U Y}\right) \sqsubseteq \mathrm{id}_{T[Y]} & \\
\Longleftrightarrow \quad p_{U Y} p_{U Y}^{\prime} r p_{U X} p_{U X}^{\sharp} r p_{U Y} p_{U Y}^{\sharp} \sqsubseteq p_{U Y} p_{U Y}^{\mathrm{H}} & \left\{p_{U Y} \text { is a function. }\right\} \\
\Longleftrightarrow \quad \theta[Y] r \theta[X] r \theta[Y] \sqsubseteq \theta[Y] & \left\{\theta[Y]=p_{U Y} p_{U Y}^{\sharp}\right\} \\
\Longleftrightarrow \quad r \theta[X] r \sqsubseteq \theta[Y] . & \{\text { Definition 3.1(a)\} }
\end{array}
$$

Following the above observation we will define the functional dependencies on attribute schemes in Dedekind categories, as follows:

DEFINITION 4.1. Let $r$ be a database relation on an attribute scheme $(U, T, \theta)$. We say that there is a functional dependency of a subset $Y$ of $U$ on a subset $X$ of $U$, denoted by $r \vDash X \rightarrow Y$, if and only if $r \theta[X] r \sqsubseteq \theta[Y]$.

We now show the basic laws on functional dependencies.

FD0. If $X \supseteq Y$, then $r \vDash X \rightarrow Y$. (Reflexive law)

Proof. Assume that $X \supseteq Y$. Then $r \theta[X] r \sqsubseteq \theta[X] \sqsubseteq \theta[Y]$, since $r \sqsubseteq \mathrm{id}_{T}$.

FD1. If $r \vDash X \rightarrow Y$ and $Z \supseteq W$, then $r \vDash X \cup Z \rightarrow Y \cup W$. (Augmentation law)

Proof. Assume that $r \theta[X] r \sqsubseteq \theta[Y]$ and $Z \supseteq W$. Then

$$
\begin{array}{rlll}
r \theta[X \cup Z] r & =r(\theta[X] \sqcap \theta[Z]) r & & \{\text { Definition 3.1(c) }\} \\
& \sqsubseteq r \theta[X] r \sqcap \theta[Z] & & \left\{r \sqsubseteq \mathrm{id}_{T}\right\} \\
& \sqsubseteq \theta[Y] \sqcap \theta[W] & & \{r \theta[X] r \sqsubseteq \theta[Y] \text { and } Z \supseteq W\} \\
& =\theta[Y \cup W] & & \{\text { Definition 3.1(c) }\} .
\end{array}
$$

FD2. If $r \vDash X \rightarrow Y$ and $r \vDash Y \rightarrow Z$, then $r \vDash X \rightarrow Z$. (Transitive law)

Proof. Assume that $r \theta[X] r \sqsubseteq \theta[Y]$ and $r \theta[Y] r \sqsubseteq \theta[Z]$. Then we have

$$
r \theta[X] r \sqsubseteq r \theta[Y] r \sqsubseteq \theta[Z] .
$$


Let $(U, T, \theta)$ be an attribute scheme in a Dedekind category $\mathcal{D}$. A formal expression $X \rightarrow Y$, namely, an ordered pair of subsets $X$ and $Y$ of $U$, joined by an arrow, is called a functional dependency. Inference rules for functional dependencies are the following three rules:

$$
\begin{array}{ll}
\text { [FD0] } & \overline{X \rightarrow Y}\{X \supseteq Y\} \\
\text { [FD1] } & \frac{X \rightarrow Y}{X \cup Z \rightarrow Y \cup W}\{Z \supseteq W\} \\
{[\text { FD2] }} & \frac{X \rightarrow Y \quad Y \rightarrow Z}{X \rightarrow Z}
\end{array}
$$

Let $F$ be a set of functional dependencies (FD's). A derivation from $F$ is a nonempty sequence

$$
\left\{X_{0} \rightarrow Y_{0}, X_{1} \rightarrow Y_{1}, \ldots, X_{n} \rightarrow Y_{n}\right\}
$$

of FD's such that, for all $k=0,1, \ldots, n$, one of the following holds:

(0) $Y_{k} \subseteq X_{k}$ ([FDol]) or $X_{k} \rightarrow Y_{k}$ is in $F$,

(i) $\exists i:: i<k$ such that

$$
\text { [FDl] } \frac{X_{i} \rightarrow Y_{i}}{X_{k} \rightarrow Y_{k}}
$$

(ii) $\exists i, j:: i, j<k$ such that

$$
\text { [FD2] } \frac{X_{i} \rightarrow Y_{i} X_{j} \rightarrow Y_{j}}{X_{k} \rightarrow Y_{k}} .
$$

For example, the union rule

$$
\text { [FD3] } \frac{X \rightarrow Y X \rightarrow Z}{X \rightarrow Y \cup Z}
$$

is proved from [FD1] and [FD2] as follows:

$$
\frac{\frac{X \rightarrow Y}{X \rightarrow X \cup Y}\{X \supseteq X\} \quad \frac{X \rightarrow Z}{X \cup Y \rightarrow Y \cup Z}\{Y \supseteq Y\}}{X \rightarrow Y \cup Z}
$$

A functional dependency $X \rightarrow Y$ is provable from $F$, written $F \vdash X \rightarrow Y$, if there is a derivation $\left\{X_{0} \rightarrow Y_{0}, X_{1} \rightarrow Y_{1}, \ldots, X_{n} \rightarrow Y_{n}\right\}$ from $F$ such that $X=X_{n}$ and $Y=Y_{n}$. A database relation $r$ is valid for $F$, written $r \models F$, if $r \models X \rightarrow Y$ is valid for all $X \rightarrow Y \in F$. Also we define a subset $F^{+}(X)$ of $U$ by $F^{+}(X)=\{a \in U \mid F \vdash X \rightarrow a\}$.

LEMMA 4.2. $F \vdash X \rightarrow Y$ iff $Y \subseteq F^{+}(X)$.

Proof. $(\Rightarrow)$ Assume that $F \vdash X \rightarrow Y$ and let $a \in Y$. Then $\vdash Y \rightarrow a$ by [FDo] and so $F \vdash X \rightarrow a$ by [FD2]. Hence $a \in F^{+}(X)$.

$(\Leftarrow)$ Assume that $Y \subseteq F^{+}(X)$. Then, for all $a \in Y$, we have $F \vdash X \rightarrow a$ by the definition of $F^{+}(X)$ and hence $F \vdash X \rightarrow Y$ by the union rule [FD3], because $U$ is a finite set. 
A family $X_{0}, W_{1}, \ldots, W_{m}$ of subsets of $U$ is a partition of $U$ if the family is disjoint and covers $U$. The set $\{1, \ldots, m\}$ will be denoted by $M$, and a union $\cup_{j \in J} W_{j}$ by $W_{J}$ for all subsets $J$ of $M$. When $J=\emptyset$, set $W_{J}=\emptyset$. Also for subsets $J, J^{\prime}$ of $M$ a set $\left(J \cap J^{\prime}\right) \cup\left(J \cup J^{\prime}\right)^{-}$will be written as $J \odot J^{\prime}$.

Definition 4.3. An attribute scheme $(U, T, \theta)$ in a Dedekind category $\mathcal{D}$ is effective if for all partitions $X_{0}, W_{1}, \ldots, W_{m}(m \geq 1)$ of $U$, there exists a family $\left\{t_{J}: I \rightarrow T \mid J \subseteq\right.$ $M$ \} of $2^{m} I$-points of $T$ indexed by all subsets of $M$ satisfying the following conditions: $\left(\mathrm{a}_{m}\right) t_{J}^{\sharp} t_{J^{\prime}} \sqsubseteq \theta\left[X_{0} \cup W_{J \odot J^{\prime}}\right]$ for all subsets $J$ and $J^{\prime}$ of $M$, $\left(b_{m}\right) t_{J}^{\sharp} t_{J^{\prime}} \sqcap \theta[\{a\}]=0_{T T}$ for all attributes $a \notin X_{0} \cup W_{J \odot J^{\prime}}$.

REMARK. The condition $\left(b_{m}\right)$ in the above definition is equivalent to a condition $\left(\mathrm{b}_{m}^{\prime}\right)$ If $Z \nsubseteq X_{0} \cup W_{J \odot J^{\prime}}$, then $t_{J}^{\|} t_{J}, \sqcap \theta[Z]=0_{T T}$.

For example, if an attribute scheme $(U, T, \theta)$ is effective, then for a subset $X_{0}$ of $U$ there is a pair of $I$-points $s, t: I \rightarrow T$ satisfying the following conditions:

(a) $s^{\sharp} t \subseteq \theta\left[X_{0}\right]$

(b ) $s^{\natural} t \sqcap \theta[\{a\}]=0_{T T}$ for all attributes $a \notin X_{0}$.

Proposition 4.4. Assume that $(U, T, \theta)$ is effective and $X_{0}$ is a subset of $U$. and a given subset $X_{0}$ of $U$, a pair $s, t: I \rightarrow T$ of $I$-points of $T$ satisfies the above conditions $\left(a_{1}\right)$ and $\left(b_{1}\right)$. Define a database relation $\tau_{0} \sqsubseteq \mathrm{id} T$ by $r_{0}=s^{\sharp} \sqcup t^{\sharp} t$. Then for all subsets $Z$ of $U$ the following hold:

(a) $Z \subseteq X_{0}$ if and only if $r_{0}=\emptyset \rightarrow Z$,

(b) If $Z \nsubseteq X_{0}$, then $r_{0} \vDash Z \rightarrow U$.

Proof. (a) Note that $r_{0} \nabla_{T I}=\left(s^{\sharp} s \cup t^{\sharp} t\right) \nabla_{T I}=s^{\sharp} \sqcup t^{\sharp}$ by the totality $s \nabla_{T I}=$ $\nabla_{I I}=\mathrm{id}_{I}$ and that

$$
r_{0} \nabla_{T T} r_{0}=r_{0} \nabla_{T I} \nabla_{I T} T_{0}=\left(s^{\sharp} \sqcup t^{\sharp}\right)(s \sqcup t)=s^{\sharp} s \sqcup s^{\sharp} t \sqcup t^{\sharp} s \sqcup t^{\sharp} t .
$$

Hence it is easy to see that $s^{\sharp} t \sqsubseteq \theta[Z]$ iff $r_{0} \nabla_{T T} r_{0} \sqsubseteq \theta[Z]$, which is equivalent to $r_{0}=\emptyset \rightarrow Z$. Thus it is enough to show that $s^{\sharp} t \subseteq \theta[Z]$ iff $Z \subseteq X_{0}$. Assume that $Z \subseteq X_{0}$. Then $s^{\sharp} t \sqsubseteq \theta\left[X_{0}\right] \sqsubseteq \theta[Z]$ by the condition (a $a_{1}$ and Lemma 3.3(a). Conversely assume that $s^{\sharp} t \sqsubseteq \theta[Z]$. Then for all $a \in Z$ we have $s^{\sharp} t \sqsubseteq \theta[\{a\}]$ by Lemma 3.3(a) and so $a \in X_{0}$, unless $s^{\sharp} t \sqcap \theta[\{a\}]=0_{T T}$ by the property of the pair $(s, t)$. (Note that $s^{\sharp} t \neq 0_{T T}$ because $s s^{\sharp} t t^{\sharp}=\mathrm{id}_{I} \neq 0_{I I}$ )

(b) Assume that $Z \nsubseteq X_{0}$. Then there is at least one attribute $a \in Z$ such that $a \notin X_{0}$. It follows from $s^{\sharp} t \sqcap \theta[\{a\}]=0_{T T}$ and $\theta[Z] \sqsubseteq \theta[\{a\}]$ that $s \theta[Z] t^{\sharp}=0_{I I}$ by Lemma $2.2(b)$ and so

$$
r_{0} \theta[Z] r_{0}=\left(s^{\sharp} s \sqcup t^{\sharp} t\right) \theta[Z]\left(s^{\sharp} s \sqcup t^{\sharp} t\right)=s^{\sharp} s \theta[Z] s^{\sharp} s \sqcup t^{\sharp} t \theta[Z] t^{\sharp} t=s^{\sharp} s \sqcup t^{\sharp} t \sqsubseteq \operatorname{id}_{T}=\theta[U],
$$

since $s^{\mathbf{t}} s \sqsubseteq \mathrm{id}_{T} \sqsubseteq \theta[Z]$.

Now we will state the soundness and the completeness theorems of functional dependencies for database relations in Dedekind categories. 
THEOREM 4.5. If a relational scheme $(U, T, \theta)$ in a Dedekind category $\mathcal{D}$ is effective, then it is sound and complete, that is, for all sets $F$ of FD's

$$
F \vdash X \rightarrow Y \Longleftrightarrow[\forall r: r \vDash F \Rightarrow r \vDash X \rightarrow Y] .
$$

Proof. (1) First we show the soundness

$$
F \vdash X \rightarrow Y \Longrightarrow[\forall r: r \vDash F \Rightarrow r \vDash X \rightarrow Y] \text {. }
$$

Assume that $F \vdash X \rightarrow Y$ and $r \models F$. Then it is trivial $r \vDash X \rightarrow Y$, since the relational database model satisfies the basic laws FD0, FD1 and FD2.

(2) Next we show the completeness

$$
[\forall r: r \vDash F \Rightarrow r \vDash X \rightarrow Y] \Longrightarrow F \vdash X \rightarrow Y
$$

Assume that $[\forall r: r \vDash F \Rightarrow \dot{r} F X \rightarrow Y]$. By the effectivity of the relational scheme $(U, T, \theta)$, one can choose a pair of $I$-points $s, t: I \rightarrow T$ of $T$ such that $s^{\mathbb{t}} t \sqsubseteq \theta\left[F^{+}(X)\right]$ and $s^{\sharp} t \sqcap \theta[\{a\}]=0_{T T}$ for $a \notin F^{+}(X)$. Set $r_{0}=s^{\sharp} s \sqcup t^{\sharp} t\left(\sqsubset \mathrm{id}_{T}\right)$. Then we will show $r_{0} \vDash F$, that is, $r_{0} \vDash Z \rightarrow W$ for all $Z \rightarrow W \in F$.

(i) In the case of $Z \subseteq F^{+}(X)$. Then $F \vdash X \rightarrow Z$ by Lemma 4.2. As $Z \rightarrow W \in F$ we have $F \vdash X \rightarrow W$ using [FD2] and so $W \subseteq F^{+}(X)$. Hence $r_{0} \vDash \emptyset \rightarrow W$ by Proposition 4.4(a). Recall that $r_{0} \vDash Z \rightarrow$ always holds by the reflexive law FDo. Therefore $r_{0} \vDash Z \rightarrow W$ by FD2.

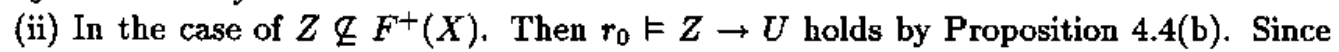
$r_{0} \vDash U \rightarrow W$ always holds by FD0, we have $r_{0} \vDash Z \rightarrow W$ using FD2.

By the assumption, we have $r_{0} \vDash X \rightarrow Y$. Then we will see $F \vdash X \rightarrow Y$. Since $X \subseteq F^{+}(X)$, we have $r_{0} \vDash \emptyset \rightarrow X$ by Proposition 4.4(a). Hence $r_{0} \vDash \emptyset \rightarrow Y$ by FD2, which is equivalent to $Y \subseteq F^{+}(X)$ by Proposition 4.4(a). We conclude $F \vdash X \rightarrow Y$ by Lemma 4.2.

\section{Multivalued Dependencies}

Let $r$ be a database relation on an attribute scheme $(U, T[U], \theta)$ discussed in Section 2. The original definition of the multivalued dependency in Beeri et al. (1977) will be transformed as follows (Set $Z=\neg(X \cup Y)$ ):

$$
\begin{aligned}
& r p_{U X} p_{U X}^{\sharp} r p_{U Y}=r p_{U X \cup Z} p_{U X \cup Z}^{\sharp} r p_{U Y} \\
& \Longleftrightarrow r p_{U X} p_{U X}^{\sharp} r p_{U Y} p_{U Y}^{\sharp}=r p_{U X \cup Z} p_{U X \cup Z}^{\sharp} r p_{U Y} p_{U Y}^{\sharp} \quad\left\{p_{U Y} p_{U Y}^{\sharp} p_{U Y}=p_{U Y}\right\} \\
& \Longleftrightarrow r \theta[X] r \theta[Y]=r \theta[X \cup Z] r \theta[Y] \\
& \Longleftrightarrow r \theta[X] r \theta[Y] \sqsubseteq r \theta[X \cup Z] r \theta[Y] \\
& \Longleftrightarrow r \theta[X] r \sqsubseteq \theta[X \cup Z] r \theta[Y] \\
& \Longleftrightarrow r \theta[X] r \sqsubseteq \theta[X \cup \neg Y] r \theta[Y]
\end{aligned}
$$

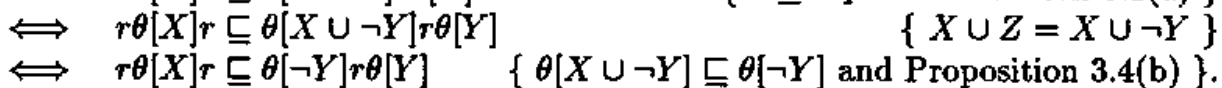

$$
\begin{aligned}
& \left\{\theta[X]=p_{U X} p_{U X}^{\sharp}\right\} \\
& \left\{r \sqsubset \mathrm{id}_{T} \text { and Definition } 3.1(\mathrm{a})\right\}
\end{aligned}
$$

We now define multivalued dependencies on attribute schemes in Dedekind categories, as follows:

Definition 5.1. Let $r$ be a database relation on an attribute scheme $(U, T, \theta)$. We say that there is a multivalued dependency of a subset $Y$ of $U$ on a subset $X$ of $U$, denoted by $r \vDash X \rightarrow Y$, if and only if $r \theta[X] r \sqsubseteq \theta[\neg Y] r \theta[Y]$. 
First of all we show the basic laws for multivalued dependencies.

MVDo. If $X \supseteq Y$, then $r \vDash X \rightarrow Y$. (Reflexive law)

Proof. Assume that $X \supseteq Y$. Then $r \theta[X] r \sqsubseteq r \theta[X] \sqsubseteq r \theta[Y] \sqsubseteq \theta[\neg Y] r \theta[Y]$, since $r \sqsubseteq \mathrm{id}_{T}$ and $\mathrm{id}_{T} \sqsubseteq \theta[\neg Y]$. law)

MVD1. If $r \models X \rightarrow Y$ and $Z \supseteq W$, then $r \models X \cup Z \rightarrow Y \cup W$. (Augmentation

Proof. Assume that $r \theta[X] r \sqsubseteq \theta[\neg Y] r \theta[Y]$ and $Z \supseteq W$. Then

$$
\begin{array}{rlll}
r \theta[X \cup Z] r & =r(\theta[X] \cap \theta[Z]) r & & \{\text { Definition 3.1(c) }\} \\
& \sqsubseteq r \theta[X] r \sqcap \theta[Z] & & \left\{r \sqsubseteq \text { id }_{T}\right\} \\
& \sqsubseteq \theta[\neg Y] r \theta[Y] \cap \theta[Z] & & \{r \theta[X] r \sqsubseteq \theta[\neg Y] r \theta[Y]\} \\
& \sqsubseteq \theta[\neg Y] r \theta[Y \cup Z] & & \{\text { Proposition 3.4(b) }\} \\
& \sqsubseteq \theta[\neg Y \cap \neg W] r \theta[Y \cup W] & \{\neg Y \supseteq \neg Y \cap \neg W \text { and } Z \supseteq W\} .
\end{array}
$$

MVD2. If $r \models X \rightarrow Y$ and $r \models Y \rightarrow Z$, then $r \models X \rightarrow Z \cap \neg Y$. (Transitive law)

Proof. Assume that $r \theta[X] r \sqsubseteq \theta[\neg Y] r \theta[Y]$ and $r \theta[Y] r \sqsubseteq \theta[\neg Z] r \theta[Z]$. First note that $r \theta[X] r \subseteq r \theta[Y] r \theta[\neg Y]$ and $r \theta[Y] r \subseteq r \theta[\neg Z \cup Y] r \theta[Z]$. Then

$$
\begin{array}{rll}
r \theta[X] r & \sqsubseteq & r \theta[Y] r \theta[\neg Y] \\
& \sqsubseteq & \theta[\neg Z \cup Y] r \theta[Z] \theta[\neg Y] \\
& =\theta[\neg Z \cup Y] r \theta[Z \cap \neg Y] .
\end{array}
$$

MVD3. $r \vDash X \rightarrow Y$ if and only if $r \vDash X \rightarrow \neg Y$. (Complement law)

Proof. Assume that $r \theta[X] r \sqsubseteq \theta[\neg Y] r \theta[Y]$. Since $r^{\sharp}=r$ and $\theta[Y]^{\sharp}=\theta[Y]$, we have $r \theta[X] r=(r \theta[X] r)^{\sharp} \sqsubseteq(\theta[\neg Y] r \theta[Y])^{\sharp}=\theta[Y] r \theta[\neg Y]$.

FD-MVD1. If $r \vDash X \rightarrow Y$, then $r \vDash X \rightarrow Y$.

Proof. Assume that $r \theta[X] r \sqsubseteq \theta[Y]$. Then $r \theta[X] r \sqsubseteq r \theta[Y] \sqsubseteq \theta[\neg Y] r \theta[Y]$ by $\mathrm{id}_{T} \sqsubseteq \theta[\neg Y]$.

FD-MVD2. Let $Y \cap Z=\emptyset$ and $Y \supseteq W$. If $r \vDash X \rightarrow Y$ and $r \vDash Z \rightarrow W$, then $\tau \vDash X \rightarrow W$.

Proof. Assume that $Y \cap Z=\emptyset, Y \supseteq W, r \theta[X] r \sqsubseteq \theta[\neg Y] r \theta[Y]$ and $r \theta[Z] r \sqsubseteq$ $\theta[W]$. Then

$$
\begin{array}{rlll}
r \theta[X] r & \sqsubseteq & r \theta[\neg Y] r \theta[Y] & \{r \theta[X] r \sqsubseteq \theta[\neg Y] r \theta[Y]\} \\
& \sqsubseteq \quad r \theta[Z] r \theta[Y] & \{\theta[\neg Y] \sqsubseteq \theta[Z] \text { by } Y \cap Z=\theta\} \\
& \sqsubseteq \quad \theta[W] \theta[Y] & \{r \theta[Z] r \sqsubseteq \theta[W]\} \\
& \sqsubseteq \theta[W] \theta[W] & \{Y \supseteq W\} \\
& \sqsubseteq \theta[W] . &
\end{array}
$$


The next proposition indicates the well-known fact that multivalued dependency is a special case of join dependency. The proof follows from Lemma 3.3(b), and is omitted.

Proposition 5.2. Let $r$ be a database relation on an attribute scheme $(U, T, \theta)$. Then for subsets $X$ and $Y$ of $U$ the following two conditions are equivalent:

(a) $r \theta[X] r \sqsubseteq \theta[\neg Y] r \theta[Y]$,

(b) $\theta[X \cup Y] r \theta[X \cup Y] \sqcap \theta[X \cup \neg Y] r \theta[X \cup \neg Y] \sqsubseteq r$.

Let $(U, T, \theta)$ be an attribute scheme in a Dedekind category $\mathcal{D}$. A formal expression $X \rightarrow Y$, namely, an ordered pair of subsets $X$ and $Y$ of $U$, joined by a two-head arrow, is called a multivalued dependency. The inference system for multivalued dependency consists of the following six rules:

$$
\begin{aligned}
\text { [MVD0] } & \frac{X \rightarrow Y}{X \rightarrow X \supseteq Y\}} \\
\text { [MVD1] } & \frac{X \rightarrow Y}{X \cup W \rightarrow Y \cup Z}\{W \supseteq Z\} \\
\text { [MVD2] } & \frac{X \rightarrow Y Y Y \rightarrow Z}{X \rightarrow Z \cap \neg Y} \\
\text { [MVD3] } & \frac{X \rightarrow Y}{X \rightarrow \neg Y} \\
\text { [FD-MVD1] } & \frac{X \rightarrow Y}{X \rightarrow Y} \\
& \frac{X \rightarrow Y \quad Z \rightarrow W}{X \rightarrow W}\{Y \cap Z=\emptyset, Y \supseteq W\}
\end{aligned}
$$

The following inference rules [MVD4] and [MVD5], called the union and the intersection rules respectively, were derived from the inference rules [MVD0]-[MVD3] in Mendelzon (1979).

$$
\begin{array}{ll}
\text { [MVD4] } & \frac{X \rightarrow Y X \rightarrow Z}{X \rightarrow Y \cup Z} \\
\text { [MVD5] } & \frac{X \rightarrow Y X \rightarrow Z}{X \rightarrow Y \cap Z}
\end{array}
$$

In the rest of this section we will prove some properties of a particular database relation, which will be needed for formalizing the proof originally given in Beeri et al. (1977) of completeness theorem for the inference rules [FD0]-[FD2], [MVD0]-[MVD3] and [FDMVD1]-[FD-MVD2].

Let $X_{0}, W_{1}, \ldots, W_{m}(m \geq 1)$ be a partition of $U$, and assume that a family $\left\{t_{J}\right.$ : $I \rightarrow T \mid J \subseteq M\}$ of $2^{m} I$-points of $T$ indexed by all subsets of $M$ satisfies the following conditions:

$\left(a_{m}\right) t_{J}^{\sharp} t_{J^{\prime}} \sqsubseteq \theta\left[X_{0} \cup W_{J \odot J^{\prime}}\right]$ for all subsets $J$ and $J^{\prime}$ of $M$, $\left(b_{m}\right) t_{J}^{\|} t_{J^{\prime}} \sqcap \theta[\{a\}]=0_{T T}$ for all attributes $a \notin X_{0} \cup W_{J \odot J^{\prime}}$.

Then define a particular database relation $r_{0}: T \neg T$ by $r_{0}=\sqcup_{J \subseteq M} t_{J}^{\sharp} t_{J}$. 
REMARK. If $t_{J}^{\sharp} t_{J^{\prime}} \sqsubseteq \theta[Z]$, then $t_{J}^{\sharp} t_{J^{\prime}} \sqsubseteq r_{0} \theta[Z] r_{0}$ as follows: Assume that $t_{J^{\prime}}^{\|} t_{J^{\prime}} \sqsubseteq$ $\theta[Z]$. Then $t_{J} \theta[Z] t_{J^{\prime}}^{\sharp}=\mathrm{id}_{I}$ by Lemma $2.2(\mathrm{a})$, and so $t_{J}^{\sharp} t_{J^{\prime}}=t_{J}^{\sharp} t_{J} \theta[Z] t_{J^{\prime}}^{\sharp} t_{J^{\prime}} \sqsubseteq r_{0} \theta[Z] r_{0}$.

LEMMA 5.3. (a) $r_{0} \vDash Z \rightarrow X_{0}$ for all subsets $Z$ of $U$,

(b) $r_{0} \vDash\{a\} \rightarrow W_{i}$ for all $a \in W_{i}$,

(c) $r_{0} \not \neq \neg W_{i} \rightarrow W_{i}$,

(d) $r_{0} \vDash Z \rightarrow W_{i}$ for all subsets $Z$ of $U$,

(e) $r_{0} \not \forall \neg W_{i} \rightarrow W$ for all nonempty proper subsets $W$ of $W_{i}$.

Proof. (a) It is trivial by $\left(\mathrm{a}_{m}\right)$ that $r_{0} \nabla_{T T} r_{0}=\sqcup_{J, J^{\prime} \subseteq M^{\prime}} t_{J^{\prime}} t_{J^{\prime}} \subseteq \theta\left[X_{0}\right]$.

(b) Assume that $a \in W_{i}$. If $i \in J \odot J^{\prime}$, then $t_{J}^{*} t_{J^{\prime}} \sqsubseteq \theta\left[X_{0} \cup W_{J \odot J^{\prime}} \subseteq \theta[\{a\}]\right.$ by (am and so $t_{J} \theta[\{a\}] t_{J^{\prime}}^{\sharp}=\mathrm{id}_{I}$ by Lemma 2.2(a). Moreover, if $i \notin J \odot J^{\prime}$, then $a \notin X_{0} \cup W_{J_{\odot} J^{\prime}}$ and so $t_{J} \theta[\{a\}] t_{J}^{\sharp}=0_{I I}$ by $\left(b_{m}\right)$ and Lemma $2.2(b)$. Hence

$$
r_{0} \theta[\{a\}] r_{0}=\sqcup_{i \in J \odot J^{\prime}} t_{J}^{\sharp} t j^{\prime} \sqsubseteq \theta\left[W_{i}\right] .
$$

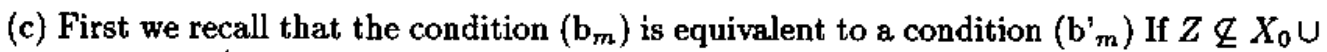
$W_{J \odot J^{\prime}}$, then $t_{J}^{\sharp} t_{J^{\prime}} \cap \theta[Z]=0_{T T}$. Assume that $r_{0} \vDash \neg W_{i} \rightarrow W_{i}$, that is, $r_{0} \theta\left[\neg W_{i}\right] r_{0} \sqsubseteq$ $\theta\left[W_{i}\right]$. Now choose $J=M$ and $J^{\prime}=M-\{i\}$. (Note that $M \odot(M-\{i\})=M-\{i\}$.) Then we have $t_{M}^{\sharp} t_{M-\{i\}} \sqsubseteq r_{0} \theta\left[\neg W_{i}\right] r_{0} \sqsubseteq \theta\left[W_{i}\right]$, since $t_{M}^{\sharp} t_{M-\{i\}} \sqsubseteq \theta\left[X_{0} \cup W_{M-\{i\}}\right]=\theta\left[\neg W_{i}\right]$ by $\left(a_{m}\right)$. On the other hand it follows from $\left(b_{m}^{\prime}\right)$ that $t_{M}^{\sharp} t_{M-\{i\}} \sqcap \theta\left[W_{i}\right]=0_{T T}$, which is a contradiction.

(d) Since $r_{0} \nabla_{T_{I}}=\cup_{J \subseteq M} t_{J}^{\sharp}$ by the totality of $t_{J}$ 's, we have

$$
r_{0} \nabla_{T T} r_{0}=r_{0} \nabla_{T I} \nabla_{I T} r_{0}=\left(\cup_{J \subseteq M} t_{J}^{\sharp}\right)\left(\omega_{J \subseteq M} t_{J}\right)
$$

Hence to prove that $r_{0} \nabla_{T T} r_{0} \sqsubseteq r_{0} \theta\left[\neg W_{i}\right] r_{0} \theta\left[W_{i}\right] r_{0}$ it suffices to see that $t_{J}^{\sharp} t_{J^{\prime}} \subseteq$ $r_{0} \theta\left[\neg W_{i}\right] r_{0} \theta\left[W_{i}\right] r_{0}$ for all $J_{,} J^{\prime} \subseteq M$. First assume that $i \in J \odot J^{\prime}$. Then $t_{j}^{\prime} t_{J^{\prime}} \sqsubseteq$ $\theta\left[X_{0} \cup W_{J \odot J^{\prime}}\right] \sqsubseteq \theta\left[W_{i}\right]$. Recall that it is trivial that $t_{J}^{\sharp} t_{J} \sqsubseteq \theta\left[\neg W_{i}\right]$. Hence we have

$$
t_{J}^{\sharp} t_{J^{\prime}}=\left(t_{J}^{\sharp} t_{J}\right)\left(t_{J}^{\sharp} t_{J^{\prime}}\right) \sqsubseteq r_{0} \theta\left[\neg W_{\mathrm{i}}\right] r_{0} \theta\left[W_{i}\right] r_{0} .
$$

Next assume that $i \notin J$ and $i \in J^{\prime}$. (Note that $i \notin J \odot J^{\prime}$ iff $i \notin J$ and $i \in J^{\prime}$, or $i \in J$ and $i \notin J^{\prime}$.) Set $J^{\prime \prime}=J \cup\{i\}$. Then it is easy to see that $J \odot J^{\prime \prime}=M-\{i\}$ and $J^{\prime \prime} \odot J^{\prime}=\left(J \odot J^{\prime}\right) \cup\{i\}$. Hence using $\left(a_{m}\right)$ we have

$$
t_{J}^{\sharp} t_{J^{\prime \prime}} \sqsubseteq \theta\left[X_{0} \cup W_{M-\{i\}}\right]=\theta\left[\neg W_{i}\right]
$$

and

$$
t_{J^{\prime}}^{\sharp} t_{J^{\prime}} \sqsubseteq \theta\left[X_{0} \cup W_{J \odot J^{\prime}} \cup W_{i}\right] \sqsubseteq \theta\left[W_{i}\right]
$$

which shows that $t_{J}^{\sharp} t_{J^{\prime}}=t_{J}^{\sharp} t_{J^{\prime \prime}} t_{J^{\prime \prime}} t_{J^{\prime}} \sqsubseteq r_{0} \theta\left[\neg W_{i}\right] r_{0} \theta\left[W_{i}\right] r_{0}$.

(e) Assume that $r_{0} \models \neg W_{i} \rightarrow W$ for a nonempty proper subsets $W$ of $W_{i}$, that is, $r_{0} \theta\left[\neg W_{i}\right] r_{0} \sqsubseteq \theta[\neg W] r_{0} \theta[W]$. Now choose $J=M$ and $J^{\prime}=M-\{i\}$. Then, since $t_{M}^{\sharp} t_{M-\{i\}} \sqsubseteq \theta\left[\neg W_{i}\right]$ in the same way as in (c), we have

$$
t_{M}^{\sharp} t_{M-\{i\}} \sqsubseteq r_{0} \theta\left[\neg W_{i}\right] r_{0} \sqsubseteq \theta[\neg W] r_{0} \theta[W]
$$


and so $t_{M} \theta[\neg W] r_{0} \theta[W] t_{M-\{i\}}^{\sharp}=\mathrm{id}_{I}$ by Lemma 2.2(a). To show that the last fact is a contradiction we will see that $t_{M} \theta[\neg W] r_{0} \theta[W] t_{M-\{i\}}^{\sharp}=0_{I I}$. Assume that $J$ is a subset of $M$ containing $i$. Since $J \odot(M-\{i\})=J-\{i\}$ and $W \subset W_{i} \subseteq \neg\left(X_{0} \cup W_{J-\{i\}}\right)$, it follows from $\left(\mathrm{b}_{m}^{\prime}\right)$ that $t_{J} \theta[W] t_{M-\{i\}}^{\forall}=0_{I I}$. Next assume that $i \notin J$. Since $M \odot J=J$ and $\emptyset \neq \neg W \cap W_{i} \subseteq \neg W \cap \neg\left(X_{0} \cup W_{J}\right)$, it follows from ( $\left.b_{m}^{\prime}\right)$ that $t_{M} \theta[\neg W] t_{J}^{\prime \prime}=0_{I I}$. Therefore

$$
\begin{aligned}
t_{M} \theta[\neg W] r_{0} \theta[W] t_{M-\{i}^{\sharp} & =\sqcup_{J \subseteq M} t_{M} \theta[\neg W] t_{J}^{\sharp} t_{J} \theta[W] t_{M-\{i\}}^{\sharp} \\
& =0_{I I} .
\end{aligned}
$$

Corollary 5.4. (a) If $\emptyset \neq W \subseteq W_{i}$, then $r_{0}=Z \rightarrow W$ if and only if $Z \cap W_{i} \neq$ b,

(b) If $\emptyset \neq W \subset W_{i}$, then $r_{0} \vDash Z \rightarrow W$ if and only if $Z \cap W_{i} \neq \emptyset$.

Proof. (a) Let $\emptyset \neq W \subseteq W_{i}$. First assume that $Z \cap W_{i} \neq \emptyset$, that is, there exists $a \in Z \cap W_{i}$. Then, recalling $r_{0} \models\{a\} \rightarrow W_{i}$ in Lemma 5.3(b) and applying the laws FD0, FD1 and FD2 a dependency $r_{0} \vDash Z \rightarrow W$ follows. Conversely assume that $Z \cap W_{i}=\emptyset$, that is, $Z \subseteq \neg W_{i}$. Notice that $r_{0}=W \rightarrow W_{i}$ is valid by Lemma $5.3(\mathrm{~b})$. If $r_{0} \models Z \rightarrow W$ is valid, we immediately have $r_{0} \models \neg W_{i} \rightarrow W_{i}$ again using FD2 and FD1. However this contradicts a fact $r_{0} \not \neg W_{i} \rightarrow W_{i}$ already seen in Lemma 5.3(c). Hence $r_{0} \not \neq Z \rightarrow W$.

(b) Let $\emptyset \neq W \subset W_{i}$. First assume that $Z \cap W_{i} \neq \emptyset$. Then $r_{0} \vDash Z \rightarrow W$ is valid by (a) and so $r_{0}=Z \rightarrow W$ by using the law FD-MVD1. Conversely assume that $Z \cap W_{i}=\emptyset$, that is, $Z \subseteq \neg W_{i}$. If $r_{0} \vDash Z \rightarrow W$ is valid, a dependency $r_{0} \vDash \neg W_{i} \rightarrow W$ follows from the augmentation law MVD1. But this is impossible by Lemma 5.3(e). Therefore $r_{0} \not \models Z \rightarrow W$.

\section{References}

Armstrong, W.W. (1974). Dependency structures of data base relationships, Proc. of IFIP '74, North Holland, 580-583.

Beeri, C., Fagin, R. and Howard, J.H. (1977). A complete axiomatization for functional and multivalued dependencies in database relations, in: Proc. ACM SIGMOD Internat. Conf. on Management of Data, Toronto, 1977 (ACM, New York, 1983), 47-61.

Bhattacharjee, T.K. and Mazumdar, A.K. (1998). Axiomatisation of fuzzy multivalued dependencies in fuzzy relational data model, Fuzzy Sets and Systems, 96, 343-352.

Biskup, J. (1987). On the complementation rule for multivalued dependencies in database relations, Acta Informatica, 10, 297-305.

Biskup, J. (1980). Inferences of multivalued dependencies in fixed and undetermined universes, Theoretical Computer Science, 10, 93-105.

Buszkowski, W. and Orlowska, E. (1998). Indiscernibilitiy-based formalization of dependencies in information systems, in: Orlowska,E.(ed.): Incomplete information: Rough set analysis, Physica Verlag, 293-315. 
Codd, E.F. (1970). A relational model for large shared data base relations, $C A C M, 13$, 377-387.

Desharnais, J. (1997). Monomorphic characterization of $n$-ary direct products, Prac. 3rd RelMiCS in Hammamet, Tunis, January 1997.

Freyd, P.J. and Ščedrov, A. (1990). Categories, Allegories, North-Holland.

Grant, J. and Jacobs, B.E. (1982). On the family of generalized dependency constraints, J. $A C M, \mathbf{2 9 ( 4 ) , 9 8 6 - 9 9 7 . ~}$

Kawahara, Y. (1998). Lattices in Dedekind categories, Proc. 4th RelMiCS in Warsav, Poland.

MacCaull, W. (2000a). A proof system for dependencies for information relations, Fundamenta Informaticae, 42, 1-27.

MacCaull, W. (2000b). A tableaux procedure for the implication problem for association rules, to appear in Relational Methods for Computer Science Applications, Orlowska,E. and Szalas,A.(eds.), Springer-Physica Verlag, 73-91.

Mendelzon, A.O. (1979). On axiomatizing multivalued dependencies in relational databases, $J$. $A C M, \mathbf{2 6}(1), 37-44$.

Olivier, J.-P. and Serrato, D. (1980). Catégories de Dedekind. Morphismes dans les Catégories de Schröder, C. R. Acad. Sci. Paris, 260, 939-941.

Orlowska, E. (1987). Algebraic approach to database constraints, Fundamenta Informaticae, 10, 57-68.

Ounalli, H. and Jaoua, A. (1997). Fuzzy difunctional dependencies, Proc. 3rd RelMiCS in Hammamet, Tunis, January 1997.

Schmidt, G. and Ströhlein, T. (1993). Relations and graphs - Discrete Mathematics for Computer Science - (Springer-Verlag, Berlin).

Reveived November 10, 2000

Revised Feburuary 16, 2001 